\title{
Ultimate strength of carbon nanotubes: A theoretical study
}

\author{
Qingzhong Zhao, Marco Buongiorno Nardelli, and J. Bernholc \\ Department of Physics, North Carolina State University, Raleigh, North Carolina 27695-8202
}

(Received 7 June 2001; published 27 March 2002)

\begin{abstract}
The ultimate strength of carbon nanotubes is investigated by large-scale quantum calculations. While the formation energy of strain-induced topological defects determines the thermodynamic limits of the elastic response and of mechanical resistance to applied tension, it is found that the activation barriers for the formation of such defects are much larger than estimated previously. The theoretical results indicate a substantially greater resilience and strength, and show that the ultimate strength limit of carbon nanotubes has yet to be reached experimentally. Nanotubes are indeed the strongest material known.
\end{abstract}

DOI: 10.1103/PhysRevB.65.144105

PACS number(s): 61.48.+c, 61.72.Cc, 61.72.Lk, 62.20.Fe

\section{INTRODUCTION}

The mechanical response of carbon nanotubes to severe deformations and strains has attracted much attention since their discovery in 1991. ${ }^{1,2}$ Carbon nanotubes have already demonstrated exceptional mechanical properties: ${ }^{3-6}$ their excellent flexibility during bending has been observed experimentally and studied theoretically. Nanotubes combine high stiffness with resilience and the ability to buckle and collapse in a reversible manner: even largely distorted configurations (axially compressed or twisted) can be due to elastic deformations with virtually no atomic defects involved. For these reasons, it has been suggested that carbon nanotubes could be promising candidates for a new generation of extremely light and superstrong fibers. However, experiments probing the strength of nanotubes are very challenging, due to the difficulties in growing high-quality, defect-free nanotubes of sufficient length and in measuring the strength of nanoscale objects. Theoretically, investigating the ultimate strength of carbon nanotubes requires modeling of inherently mesoscopic phenomena, such as plasticity and fracture, on a microscopic, atomistic level, which presents its own set of challenges. However, the initial stages of strain-induced transformations can be deduced from simulations and these results can be further refined by detailed investigations of the potential energy landscape.

It is now well established from simulations that beyond a certain value of the applied strain, around 5\%-6\%, singlewalled carbon nanotubes respond to the mechanical stimuli via the spontaneous formation of topological defects. ${ }^{7}$ Given the well-known $1 \mathrm{TPa}$ Young modulus of graphite and a similar modulus of nanotubes ${ }^{8}$ when rescaled to the density of graphite, the range of elastic response indicates enormous strength, unmatched by any other known material. It has also been shown that the mechanical response depends critically on tube geometry: zigzag tubes display a higher strain resistance than armchair tubes with same diameter. ${ }^{9,10}$ Different orientations of the carbon bonds with respect to the strain axis in tubes of different symmetry lead to completely different scenarios: ductile or brittle behaviors can be observed in nanotubes of different indices under the same external conditions. Furthermore, the behavior of nanotubes under large tensile strain strongly depends on their symmetry and diameter $^{7,9}$ and the initial stages of strain-induced transfor- mations can be explained by dislocation theory. ${ }^{11,7}$ Recently, some of these predictions have been confirmed by two independent experiments: a number of carbon nanotubes do not exhibit mechanical failure and breakage up to about $5 \%$ strain. ${ }^{14,15}$ These results, although important in addressing the problem of strength of carbon nanotubes, do not clearly determine the ultimate limit of their mechanical response, due to the quality of the samples and experimental limitations.

In order to determine the ultimate limits of the elastic response of strained carbon nanotubes, one needs to address not only the problem of the stability of topological defects under strain, but equally importantly, as it turns out, the energetics of the activation process. We have therefore carried out extensive large-scale quantum calculations of the mechanism of formation of strain-induced topological defects in carbon nanotubes. Our results indicate that although the topological defects become energetically stable at strains of the order of $5 \%-6 \%$, the activation barriers for their formation are extremely high, thus hindering the creation of such defects even at relatively large strains. This implies that ideal, structurally perfect single-walled carbon nanotubes should be kinetically stable and resist strains well beyond the $5 \%$ observed experimentally and that they can indeed be considered the strongest materials known.

\section{METHODOLOGY}

The quantum simulations were carried out with a multigrid-based total-energy method that uses a real-space grid as the basis. ${ }^{16}$ The Perdew-Zunger parametrization ${ }^{17}$ of the Ceperley-Alder ${ }^{18}$ exchange-correlation energy was used. The Kleinman-Bylander approach ${ }^{19}$ was chosen to include nonlocal, norm-conserving pseudopotentials. ${ }^{20-22}$ The convergence in both $\mathbf{k}$-space sampling and grid size was carefully evaluated, and the supercell containing the nanotube included a large vacuum region $(\sim 7 \AA)$, in order to ensure that the interactions between tube images can be neglected.

In order to extend this study to a larger class of systems and to overcome the computational limitations of $a b$ initio calculations, we have carried out extensive comparisons between the accurate but expensive ab initio approach and the less computationally demanding semiempirical tight-binding and classical molecular dynamics methods. The tight-binding 
and classical potential methods use adjustable parameters, which are usually determined by fitting experimental and $a b$ initio data for diamond, graphite, and carbon chains in their equilibrium configurations. The reliability of these parameters for describing the properties of strained carbon nanotube structures needs thus to be verified by full $a b$ initio calculations. In the tight-binding simulations we used an orthogonal $s p^{3}$ model of $\mathrm{Xu}$ et $a l .^{23}$ This model is able to describe the dynamical and elastic properties of nanotubes with good accuracy and reliability. The classical molecular dynamics calculations used the Tersoff-Brenner many-body potential to model interactions between carbon atoms. ${ }^{24,25}$ Although this potential is not as accurate as the quantummechanical methods, it is very useful for qualitative and semiquantitative estimates, and for explorations of the longtime evolution or of large portions of the potential energy landscape.

\section{RESULTS}

From the geometrical point of view, carbon nanotubes are hollow cylinders consisting of "rolled-up" graphite sheets (graphene). Therefore, imposing an axial tension on a nanotube is geometrically similar to imposing a lateral strain on a graphene sheet. Since the hexagons comprising the nanotube walls have different orientations with respect to the tube axis (the so-called wrapping angle), an axial tension translates into lateral strains at varying angles in the graphene sheet. In particular, an axial strain in an armchair tube corresponds to a planar tension which is applied perpendicular to the bond marked " $A B$ " in Fig. 1(a), while in a zigzag tube the planar tension is parallel to the bond indicated as " $C D$ " in Fig. 1(b). We call the tension in armchair tubes "transverse," while the one in zigzag tubes will be called "longitudinal." The transverse tension finds natural release in a $90^{\circ}$ rotation of the $A B$ bond in Fig. 1(a). This rotation changes four neighboring hexagons into two pentagons and two heptagons, forming a pentagon-heptagon-heptagon-pentagon (5-77-5) defect [indicated by dashed lines in Fig. 1(a)], and is known as the Stone-Wales transformation. ${ }^{26}$ The StoneWales transformation effectively elongates the tube in the strain direction, releasing the excess strain energy. ${ }^{7,11}$ In a zigzag tube, which experiences longitudinal strain, the $E F$ bond in Fig. 1(b) forms a $60^{\circ}$ angle with the tube axis, and a similar (5-7-7-5) defect [indicated by a dashed line in Fig. 1(b)] cannot effectively release the excess strain. This implies that a greater resistance to axial strain should be expected in zigzag nanotubes, since the onset of nonelastic behavior requires the formation of defect structures.

We have carried out extensive ab initio calculations, investigating the formation and activation energies of the (5-7$7-5)$ defect in a $(5,5)$ carbon nanotube and a graphene sheet subject to static strains of up to $15 \% .^{12}$ While the formation energy (the difference in total energies between a defective and an ideal carbon nanotube) is the quantity that determines the stability of a topological defect, the activation energy (the energy barrier that a system has to overcome to form a defect) is the quantity that determines the ultimate limit of the elastic response. If this barrier is sufficiently high, the defect
Armchair tube $(5,5)$

a)

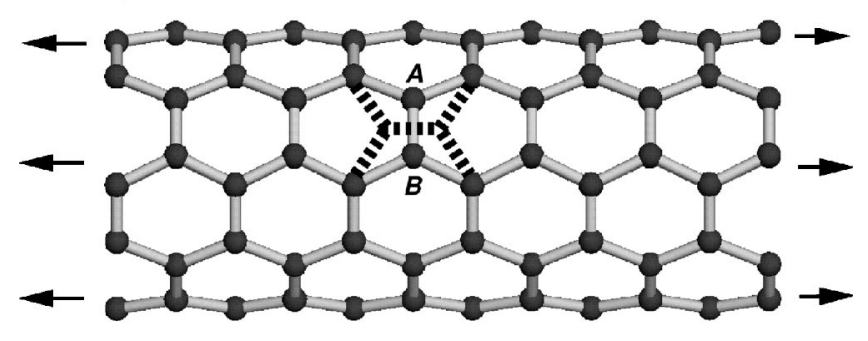

Zigzag tube $(9,0)$

b)

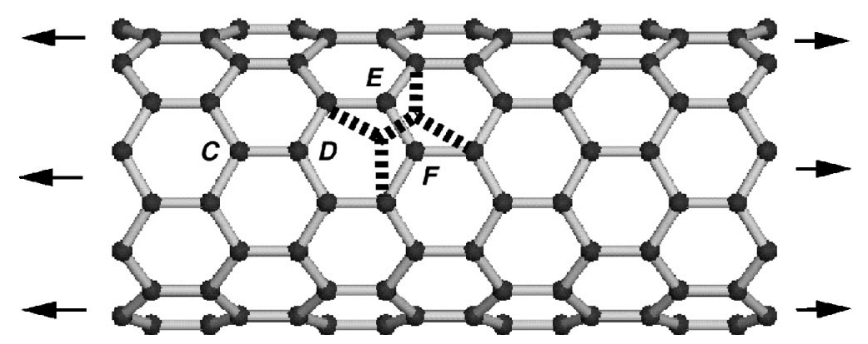

FIG. 1. Bond rotation in strained carbon nanotubes: (a) side view of a $(5,5)$ armchair tube under axial strain: rotating the bond " $A B$ " by $90^{\circ}$ forms a (5-7-7-5) defect (dashed lines). (b) Side view of a $(9,0)$ zigzag tube where the bond " $E F$ " is responsible of the formation of the (5-7-7-5) defect (dashed lines). See text.

will not be created even if its formation is thermodynamically preferred, and the elastic response will extend into the metastable regime. ${ }^{7}$ The activation barriers for the StoneWales transformation have been computed by constrained relaxation along the simplest kinetic pathway that leads to the formation of the defect. The C-C bond was rotated stepwise about its center, with only the angle of the rotation fixed at each step. The $\mathrm{C}$ atoms of the rotating bond were free to move within the plane perpendicular to the graphene sheet, and all the remaining internal degrees of freedom were fully relaxed at each step. This procedure ensured an accurate exploration of five of the six degrees of freedom of the two rotating atoms. In order to estimate the effect of the sixth degree of freedom, we also allowed the confining plane to be shifted off center in selected test calculations, which were carried out using the tight-binding method (see below). Asymmetric saddle points were indeed observed in smalldiameter nanotubes, where activation energies were reduced by a small amount (less than $0.5 \mathrm{eV}$ ) by off-center bond rotations. However, this variation is small on the energy scale of the transformation and does not affect the general interpretation of our results.

Finally, in all the above calculations we assume constantlength conditions. To ascertain the validity of this assumption we have evaluated, from tight-binding simulations, the formation energy of the 5775 defect under a constant force applied to the tube ends. For both the armchair $(5,5)$ and the zigzag $(9,0)$ tubes, the results are very similar to their constant-length counterparts. Under a constant force, the tube will undergo a sudden elongation upon the formation of 

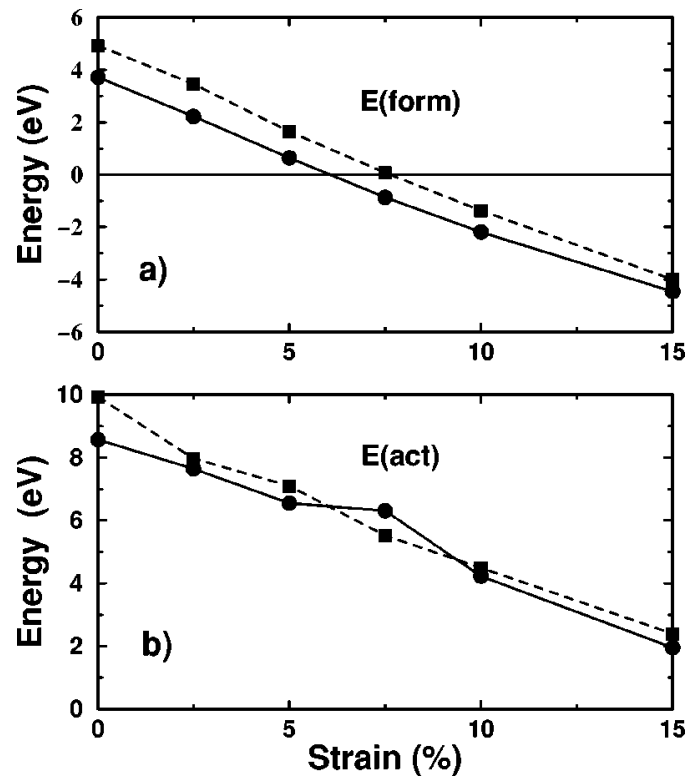

FIG. 2. (a) Formation energy of the (5-7-7-5) defect in a $(5,5)$ nanotube (circles) and a graphene sheet (squares) as function of uniaxial strain. (b) Activation energy for the formation of the (5-77-5) defect.

the defect, since the defective tube has a longer equilibrium length at a given applied force. This elongation is $\approx 0.1 \AA$ for the $(5,5)$ tube and is even smaller for the $(9,0)$ one. The presence of this discontinuity under the constant-force conditions will cause the onset of the Stone-Wales transformation to occur earlier than under constant-length conditions. From our calculations, one can estimate that the defect will be energetically favored at an elongation $0.9 \%$ smaller than at constant-length conditions. Since this change is small on the scale of the transformation, we have restricted the discussion to the constant-length conditions.

The formation energies obtained from ab initio calculations are shown in Fig. 2(a) for both the $(5,5)$ tube (solid line) and the graphene sheet (dashed line). The formation energy of the (5-7-7-5) defect decreases almost linearly with strain, and the defect becomes energetically favored at strains above 6\%. The same trend is observed for the graphene sheet, although the defect energies are about $1 \mathrm{eV}$ higher than in the $(5,5)$ tube. The lower energy of the defects in the nanotube can be attributed to its curvature, which allows for a better relaxation of bond angles. The absence of curvature in the flat graphene sheet makes the formation of the (5-7-7-5) defects less favorable, since the bond angles between the atoms comprising the defect are forced to deviate from the ideal $s p^{2}$ geometry. However, once the (5-7$7-5)$ defects are formed, their stabilities in the $(5,5)$ tube and the graphene sheet are comparable. In Fig. 2(b) we display the activation energies for defect formation in both the nanotube and sheet. These energies are very high: at $0 \%$ strain they are $8.6 \mathrm{eV}$ in the $(5,5)$ tube and $9.9 \mathrm{eV}$ in the graphene sheet. The latter value is in good agreement with the "concerted exchange" diffusion process in graphite, where a value of $10.4 \mathrm{eV}$ was obtained. ${ }^{27}$ Since the activation energies are so high, the strain relaxation process is kinetically controlled at room temperature. However, the barriers to defect formation decrease significantly with strain. For example, the activation energy for the Stone-Wales transformation in a $(5,5)$ tube becomes $1.95 \mathrm{eV}$ at $15 \%$ strain. In general, although the formation energies of the (5-7-7-5) defect in the $(5,5)$ tube are lower than those in graphene with transverse strain, the activation energies in both systems are very close.

The high activation energies suggest that the perfect hexagonal network is kinetically stable even for strain values substantially larger than 5\%. The use of a simple Arrhenius expression $\Gamma \propto N_{\text {bonds }} \bar{\nu} \exp \left(-\Delta E / k_{B} T\right)$ (Ref. 7) confirms that the tubes are extremely stable at room temperature in their hexagonal equilibrium configuration due to the large value of the activation energy $\Delta E{ }^{13}$ The same conclusion can be drawn on the basis of the only two published experiments that have measured the strength of carbon nanotubes. ${ }^{14,15}$ In both cases, some carbon nanotube samples have supported maximum strain values above 5\%. These values, although in remarkable agreement with the prediction of the thermodynamic stability of the (5-7-7-5) defect, ${ }^{7}$ do not represent the ultimate strain limit for the system. The presence of frozen-in defects that were formed during growth can certainly limit the maximum sustainable strain, since they act as nucleation centers for further topological modifications. ${ }^{7,9}$ Moreover, the appearance of a (5-7-7-5) defect can be interpreted as a nucleation of a degenerate dislocation loop in the planar hexagonal network of the graphite sheet. ${ }^{11,9}$ The configuration of this primary dipole is a (5-7) core attached to an inverted (7-5) core. The (5-7) defect behaves thus as a single-edge dislocation in the graphitic plane. Once nucleated, the (5-77-5) dislocation loop can ease further relaxation by separating the two dislocation cores, which glide through successive Stone-Wales bond rotations. This corresponds to a plastic flow of dislocations and gives rise to ductile behavior in the nanotube. Moreover, the 5-7 defect is the smallest defect that can change the tube index without drastically altering the local curvature of the nanotube. ${ }^{28-31}$ In fact, the dissociation of the (5-7-7-5) defects results in seamlessly connected nanotube sections of different symmetry. The relevance of this process for the production of all-nanotube microelectronic devices has been recently demonstrated experimentally ${ }^{32}$ and was also discussed theoretically in conjunction with adatom adsorption. ${ }^{33}$

We have extended the present investigation to a larger variety of nanotubular systems using tight-binding and classical potential methods. These more phenomenological techniques are needed to overcome the substantial limitations of $a b$ initio calculations with regard to system size and the number of cases that can be studied.

We first turn to comparisons between ab initio, tightbinding, and classical molecular dynamics results, in order to determine the reliability of the simpler techniques. Figure 3 compares the results of $a b$ initio and tight-binding calculations for both the $(5,5)$ tube and the graphene sheet. As is evident from the figure, the tight-binding results are in very good agreement with the $a b$ initio ones; e.g., the formation energies of the (5-7-7-5) defect in graphene obtained from tight-binding calculations are only $0.4 \pm 0.1 \mathrm{eV}$ higher than 

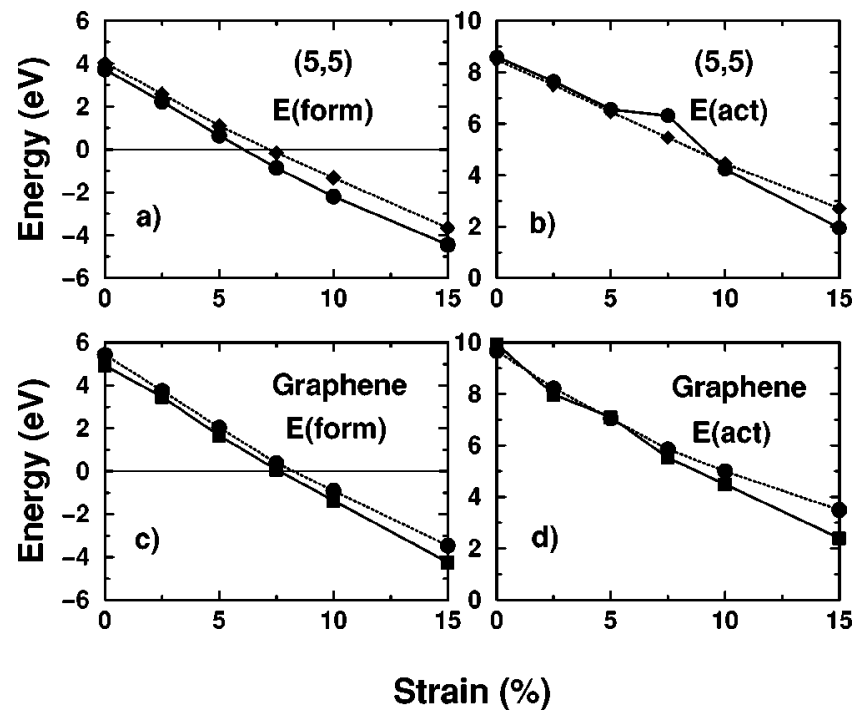

FIG. 3. Comparison between ab initio and tight-binding results for a $(5,5)$ nanotube and a graphene sheet. (a), (c) Formation energies; (b), (d) activation energies. The ab initio results are connected by a solid line, while a dashed line connects the tight-binding results.

those from $a b$ initio calculations. However, the differences between tight-binding and $a b$ initio results increase with strain. For example, the formation energies of the StoneWales defect in the $(5,5)$ tube differ by $0.1 \mathrm{eV}$ at $0 \%$ strain and by $0.7 \mathrm{eV}$ at $15 \%$ strain. This increase can be attributed to the fact that the tight-binding parameters were fitted to data for unstrained carbon systems. Therefore, their accuracy and reliability decrease with increasing strain. Moreover, the agreement between the tight-binding and $a b$ initio results is much better for graphene than for the $(5,5)$ tube. This is
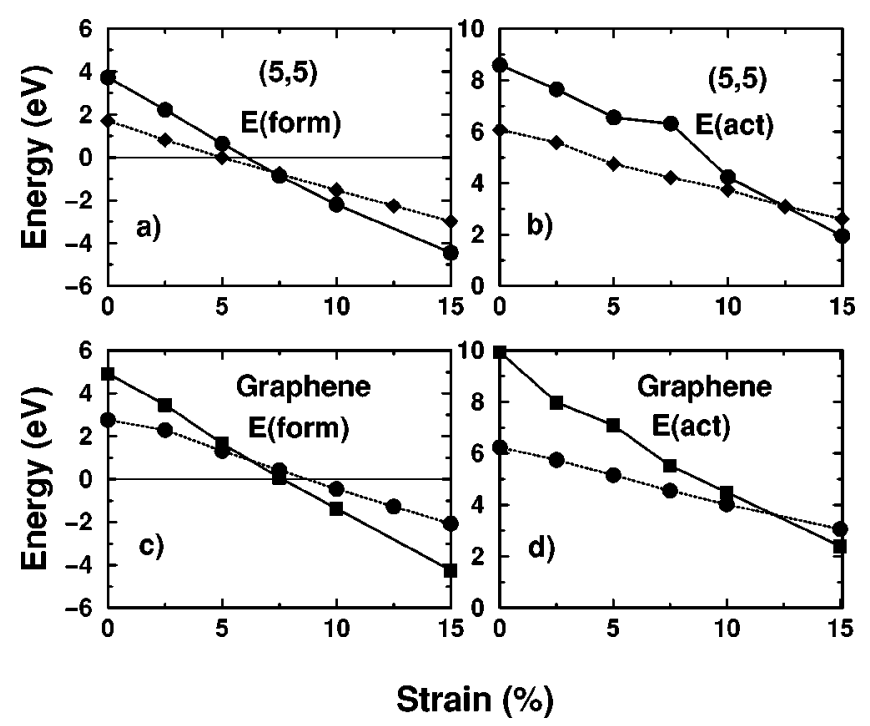

FIG. 4. Comparison between $a b$ initio and classical potential results for a $(5,5)$ nanotube and a graphene sheet. (a), (c) Formation energies; (b), (d) activation energies. The $a b$ initio results are connected by a solid line, while a dashed line connects the classical potential results.
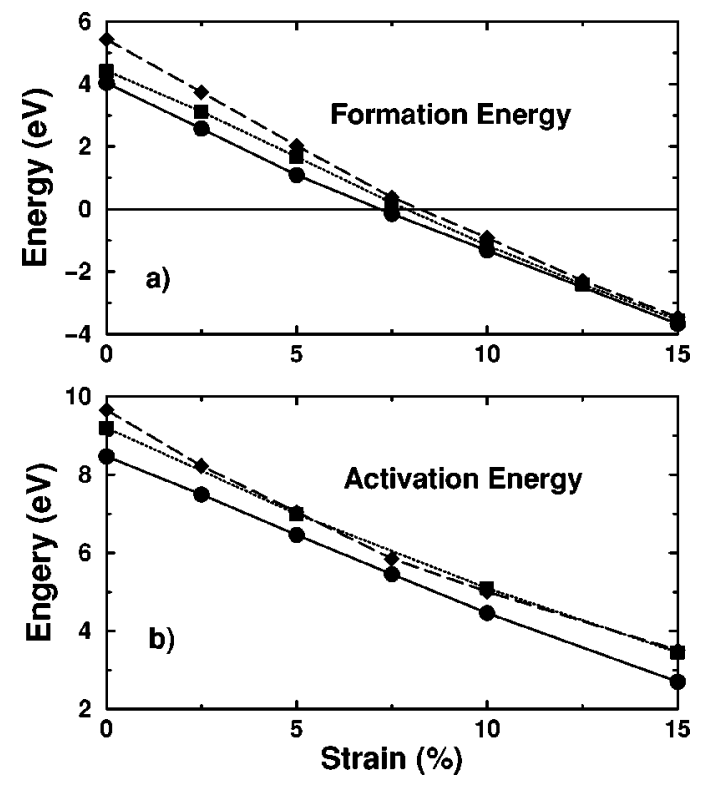

FIG. 5. Curvature effects on the formation and activation energies of the (5-7-7-5) defect in strained armchair nanotubes and graphene under transverse strain. Circles correspond to a $(5,5)$ tube, squares to a $(10,10)$ tube, and diamonds to a graphene sheet. See text.

because graphite data were used in fitting the original tightbinding parameters, while carbon nanotube data were not. In general, however, the tight-binding method is a good alternative to the computationally costly $a b$ initio method. Turning to the classical potential results, while they are able to give a qualitative and semiquantitative description of the mechanical behavior of nanotubes, they tend to underestimate the ultimate limit of the elastic response. This is shown in
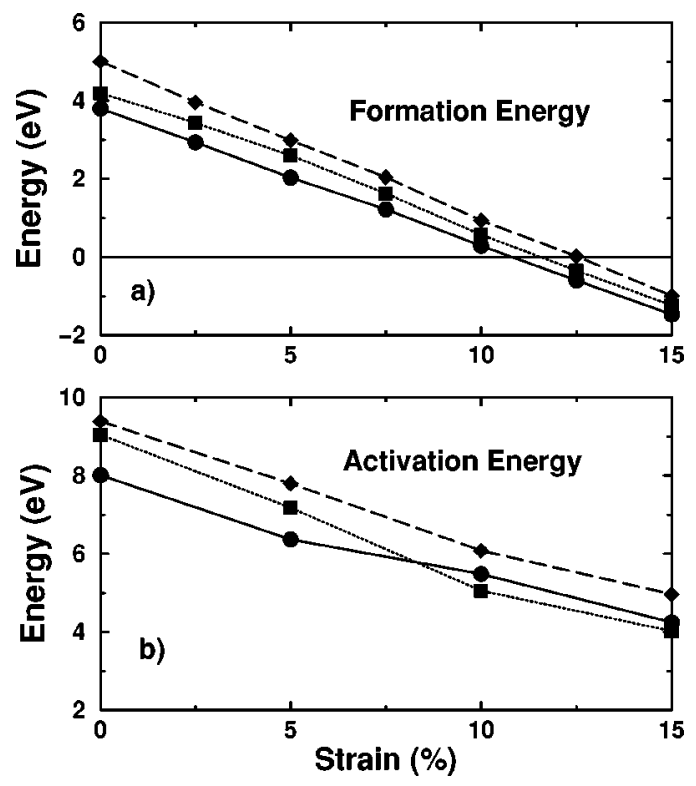

FIG. 6. Curvature effects on the formation and activation energies of the (5-7-7-5) defect in strained zigzag nanotubes and graphene under longitudinal strain. Circles correspond to a $(9,0)$ tube, squares to a $(17,0)$ tube, and diamonds to a graphene sheet. 
Fig. 4, where a comparison with ab initio results is displayed. Although the classical potentials accurately reproduce the lattice constants, the binding energies, and the elastic constants of both graphite and diamond, our comparison shows that the lack of a quantum-mechanical description leads to significant underestimates in both activation and formation energies.

We have investigated the curvature and helicity effects on the defect formation mechanism using the tight-binding method. Figures 5 and 6 show the formation and activation energies of a (5-7-7-5) defect in armchair and zigzag tubes of different diameters, and compare it to graphene. In armchair tubes, the defect becomes stable at strain values of $7 \%-8 \%$. [The ab initio result for the $(5,5)$ tubes is $6 \%$.] Furthermore, although the (5-7-7-5) defect formation is the dominant mechanism for strain release in armchair nanotubes, this is not true for zigzag tubes. ${ }^{9,10}$ From Fig. 6, this defect is thermodynamically unfavorable up to a very high strain value (over 10\%). Therefore, zigzag nanotubes are expected to be even more resilient than armchair ones and tolerate very high values of axial tension. They thus extend the limit of ultimate strength to unprecedented values, far beyond that of any other known material. The results in Figs. 5 and 6 also show the effect of curvature: the lower defect formation energy in $(5,5)$ tube suggests that $(5-7-7-5)$ defects are more favorable in smaller-diameter nanotubes. ${ }^{9}$ The curvature effect decreases with strain and seems to vanish at a high strain value.

\section{SUMMARY}

In summary, we have investigated the mechanism of strain-induced defect formation in carbon nanotubes through extensive quantum-mechanical and molecular mechanics calculations, focusing on the ultimate strength of these systems. Our results show that the excess elastic energy in a strained nanotube is released via a spontaneous formation of topological defects whose characteristics depend upon the geometry and the diameter of the nanotube. The activation energies for the defect formation are found to be significantly higher than previously estimated, which increases the theoretical predictions of the elastic response and of the onset of mechanical failure in carbon nanotubes to unprecedented values, well beyond those of other known materials. According to the theoretical estimates, carbon nanotubes are by far the strongest material known.

\section{ACKNOWLEDGMENTS}

We are pleased to acknowledge fruitful discussions with D. Orlikowski and V. Meunier. This work was supported in part by grants from NASA and ONR. We thank the DOD, NSF, and NC Supercomputing Centers for extensive computing support.
${ }^{1}$ S. Iijima, Nature (London) 354, 56 (1991).

${ }^{2}$ R.F. Service, Science 281, 940 (1998); J. Bernholc, C. Roland, and B.I. Yakobson, Curr. Opin. Solid State Mater. Sci. 2, 706 (1997); M. S. Dresselhaus, G. Dresselhaus, and P. C. Eklund, Science of Fullerenes and Carbon Nanotubes (Academic Press, San Diego, 1996); Carbon Nanotubes: Preparation and Properties, edited by T. W. Ebbesen (CRC Press, Boca Raton, 1997); R. Saito, G. Dresselhaus, and M. S. Dresselhaus, Physical Properties of Carbon Nanotubes (Imperial College Press, London, 1998).

${ }^{3}$ S. Iijima, C. Brabec, A. Maiti, and J. Bernholc, J. Chem. Phys. 104, 2089 (1996).

${ }^{4}$ B.I. Yakobson, C.J. Brabec, and J. Bernholc, Phys. Rev. Lett. 76, 2511 (1996).

${ }^{5}$ J. Despers, E. Daguerre, and K. Lafdi, Carbon 33, 87 (1995).

${ }^{6}$ N. Chopra, L. Benedict, V. Crespi, M.L. Cohen, S.G. Louie, and A. Zettl, Nature (London) 377, 135 (1995); R. Ruoff and D. Lorents, Bull. Am. Phys. Soc. 40, 173 (1995).

${ }^{7}$ M. Buongiorno Nardelli, B.I. Yakobson, and J. Bernholc, Phys. Rev. B 57, R4277 (1998).

${ }^{8}$ A. Krishan et al., Phys. Rev. B 58, 14013 (1998); D.H. Robertson, D.W. Brenner, and J.W. Mintmire, ibid. 45, 12592 (1992).

${ }^{9}$ M. Buongiorno Nardelli, B.I. Yakobson, and J. Bernholc, Phys. Rev. Lett. 81, 4656 (1998).

${ }^{10}$ P. Zhang, P.E. Lammert, and V.H. Crespi, Phys. Rev. Lett. 81, 5346 (1998).

${ }^{11}$ B.I. Yakobson, Appl. Phys. Lett. 72, 918 (1998).

${ }^{12}$ Constant-stress conditions (as opposed to constant strain) do not produce significant differences at the onset of nonelastic behavior.

${ }^{13}$ The quantities that enter in the Arrhenius expression are the activation energy for the bond rotation $\Delta E$, the absolute temperature $T$, the Boltzmann constant $k_{B}$, the number of bonds $N_{\text {bonds }}$, and an effective frequency $\bar{\nu}$, which is associated with the vibration of the bond in the direction of the saddle point. We estimate the latter using the frequency of the bond rotation mode in a carbon nanotube, $\sim 10^{13} \mathrm{sec}^{-1}$. [See, for example, J. Yu, R.K. Kalia, and P. Vashista, J. Chem. Phys. 103, 6697 (1995).]

${ }^{14}$ D.A. Walters, L.M. Ericson, M.J. Casavant, J. Liu, D.T. Colbert, K.A. Smith, and R.E. Smalley, Appl. Phys. Lett. 74, 3803 (1999).

${ }^{15}$ M.F. Yu, B.S. Files, S. Arepalli, and R.S. Ruoff, Phys. Rev. Lett. 84, 5552 (2000).

${ }^{16}$ E.L. Briggs, D.J. Sullivan, and J. Bernholc, Phys. Rev. B 52, R5471 (1995); 54, 14362 (1996).

${ }^{17}$ J.P. Perdew and A. Zunger, Phys. Rev. B 23, 5048 (1981).

${ }^{18}$ D. Ceperley and B. Alder, Phys. Rev. Lett. 45, 566 (1980).

${ }^{19}$ L. Kleinman and D. Bylander, Phys. Rev. Lett. 48, 1425 (1982).

${ }^{20}$ D. Hamann, M. Schlüter, and C. Chiang, Phys. Rev. Lett. 43, 1494 (1979).

${ }^{21}$ G. Bachelet, D. Hamann, and M. Schlüter, Phys. Rev. B 26, 4199 (1982).

${ }^{22}$ D. Hamann, Phys. Rev. B 40, 2980 (1989).

${ }^{23}$ C.H. Xu, C.Z. Wang, C.T. Chan, and K.M. Ho, J. Phys. C 4, 6047 (1992). 
${ }^{24}$ J. Tersoff, Phys. Rev. Lett. 61, 2879 (1988); Phys. Rev. B 37, 6991 (1988).

${ }^{25}$ D.W. Brenner, Phys. Rev. B 42, 9458 (1990).

${ }^{26}$ A.J. Stone and D.J. Wales, Chem. Phys. Lett. 128, 501 (1986).

${ }^{27}$ E. Kaxiras and K.C. Pandey, Phys. Rev. Lett. 61, 2693 (1988).

${ }^{28}$ B.I. Dunlap, Phys. Rev. B 46, 1933 (1992).

${ }^{29}$ T.W. Ebbesen and T. Takada, Carbon 33, 973 (1995).

${ }^{30} \mathrm{Ph}$. Lambin, A. Fonseca, J.P. Vigneron, J.B. Nagy, and A.A. Lu- cas, Chem. Phys. Lett. 245, 85 (1995).

${ }^{31}$ L. Chico, V.H. Crespi, L.X. Benedict, S.G. Louie, and M.L. Cohen, Phys. Rev. Lett. 76, 971 (1996).

${ }^{32}$ M. Ouyang, J.-L. Huang, C.L. Cheung, and C.M. Lieber, Science 291, 97 (2001).

${ }^{33}$ D. Orlikowski, M. Buongiorno Nardelli, J. Bernholc, and C. Roland, Phys. Rev. Lett. 83, 4132 (1999). 